\section{OSHA Advisory on Risks From Glass Capillary Tubes}

The FDA, the National Institute for Occupational Safety and Health (NIOSH) of the CDC, and OSHA recently issued an advisory notice on the potential risk of injury or infection from bloodborne pathogens from accidental breakage of glass capillary tubes.

Accidental breakage of slender, fragile glass capillary tubes has been reported when the tubes are inserted into putty to be sealed and during centrifugation. Breakage of the tubes may result in a penetrating wound and blood inoculation to the user. One such injury resulted in the transmission of HIV to a physician who has since died of AIDS.

To reduce the risk of injury due to breakage of capillary tubes, FDA, NIOSH, and OSHA recommend that users consider blood collection devices that are less prone to accidental breakage, including use of (1) capillary tubes that are not made of glass; (2) glass capillary tubes wrapped in puncture-resistant film; (3) products that use a method of sealing that does not require manually pushing one end of the tube into putty to form a plug, or (4) products that allow the blood hematocrit to be measured without centrifugation. The advisory notes that, although the FDA, NIOSH, and OSHA cannot recommend specific products, blood-collection devices with these characteristics are currently available, and their use may reduce the risk of injury and blood exposure. The advisory notice also summarizes requirements under the Safe Medical Devices Act (SMDA) of 1990, which requires hospitals and other user facilities to report deaths, serious illnesses, and injuries associated with the use of medical devices, including capillary tubes. Practitioners who become aware of any medical-device-related adverse event or product problem or malfunction should report it to their designated Medical Device User Facility Reporting contact person. Even if a medical-device-related incident or product quality problem is not required to be reported under the SMDA, health professionals are encouraged to report any medical-device-related concerns to MedWatch, the FDA's voluntary reporting program. Submit reports to MedWatch by telephoning 1-800-FDA-1088, by faxing 1-800-FDA-0178, via the MedWatch website, or mailing to MedWatch, FDA, HF-2, 5600 Fishers Ln, Rockville, MD 20852-9787. Copies of this advisory are available on OSHA's web site.

\section{FDA Licenses Supplemental Test for HCV}

The FDA has approved a more specific supplemental test to confirm screening-test results for antibodies to the hepatitis $\mathrm{C}$ virus (HCV). The new supplemental test, RIBA HCV 3.0 Strip Immunoblot Assay (SIA), is used to test blood specimens that already have tested repeatedly reactive on licensed screening tests. This new test can detect one more type of antibody to the HCV virus than the previous supplemental test and is better at distinguishing truly positive from falsely positive test results.
The practical and public health significance of this new supplemental test is heightened because of a recent Department of Health and Human Services recommendation to blood banks and medical facilities to notify patients who received blood from donors who subsequently were found possibly to be infected by HCV (currently, the risk of contracting HCV from a unit of blood is extremely low, approximately 1 in 100,000 units transfused). Notifications of blood recipients consistent with this recommendation are scheduled to begin no later than March 1999. The new RIBA HCV 3.0 SIA will prevent thousands of unnecessary notifications and subsequent testing and counseling of blood recipients based on inconclusive (indeterminate) $\mathrm{HCV}$ test results.

The RIBA 3.0 Strip SIA is a three-stage test that uses five genetically engineered $\mathrm{HCV}$ antigens. The previous version of the test used four antigens. In one study using a population of healthy blood donors, approximately $30 \%$ of repeatedly reactive test results were interpreted as indeterminate by the previous test. Only approximately $7 \%$ of these same results were interpreted as indeterminate by the RIBA 3.0. The RIBA 3.0 Strip SIA is manufactured by Chiron Corporation, Emeryville, California, and will be distributed by Ortho Diagnostic Systems, Inc, in Raritan, New Jersey.

FROM: Food and Drug Administration. FDA licenses improved supplemental test for hepatitis $\mathrm{C}$. News release, February 12, 1999.

\section{Faucet Aerators Source of Stenotrophomonas maltophilia}

Multiple nosocomial outbreaks have been linked to contaminated water sources within the hospital. Weber and colleagues from the University of North Carolina Hospitals reported a cluster of patients in a surgical intensive-care unit who were colonized or infected with Stenotrophomonas maltophilia.

Patients were colonized with two isolates of $S \mathrm{mal}$ tophilia, which were found by pulsed-field gel electrophoresis (PFGE) to be identical to strains isolated from the faucet aerators present in sinks in the patients' rooms. Multiple different strains, as defined by PFGE, were isolated from patients during this outbreak. The authors believe that low-level contamination of the hospital's potable water led to contamination of the faucet aerators, with subsequent bacterial amplification on the aerator, which led to contamination of water after aeration.

They suggest that cultures should be performed of faucet aerators when water sources are suspected as the reservoir for a nosocomial outbreak. If additional clusters of infected or colonized patients are linked to contaminated aerators, consideration should be given to routine disinfection or removal of the aerators.

FROM: Weber DJ, Rutala WA, Blanchet CN, Jordan M, Gergen MF. Faucet aertors: a source of patient colonization with Stenotrophomonas maltophilia. Am J Infect Control 1999;27:59. 


\section{Scrovos?}

\section{Scrub,}
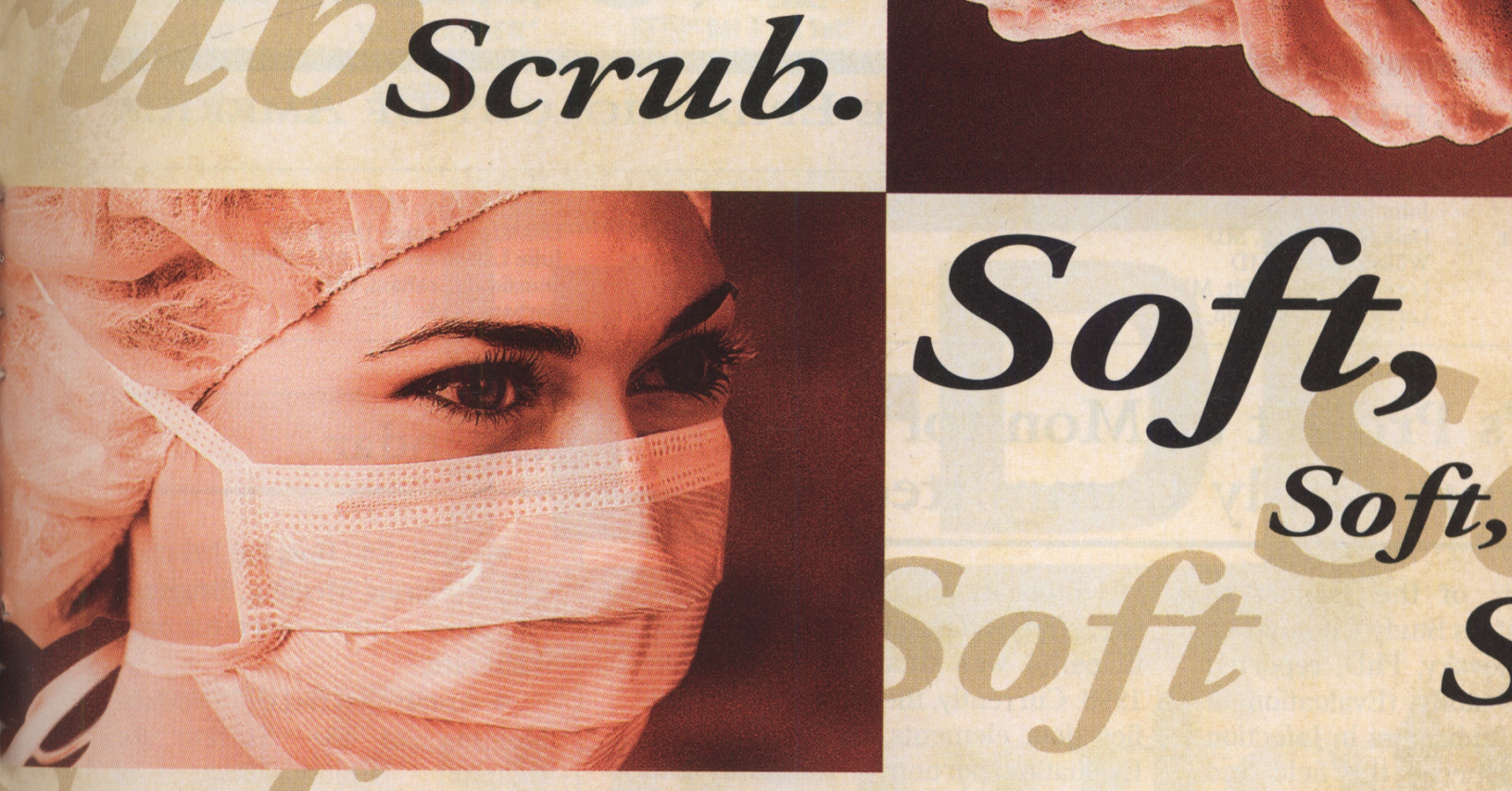

\section{New Prevacare* $S$ Surgical Scrub combines efficacy and}

gentleness to moisturize your skin between surgical procedures.

Too often harsh surgical scrubs force you to compromise between performance and skin integrity. Now Johnson \& Johnson Medical has the solution: New PREVACARE* S Surgical Scrub Antimicrobial Skin Cleanser.

PREVACARE* $^{*}$ Surgical Scrub does not even feel or smell like other traditional liquid scrub solutions. It combines the effectiveness of chlorhexidine with a patented emollient based formulation for exceptional gentleness with broad spectrum antimicrobial activity.

PREVACARE* $S$ is also completely compatible with PREVACARE* Moisturizing Lotion to ensure that CHG antimicrobial activity is maintained throughout scrub procedures. It is yet another example of the innovation and excellence you can expect from the complete line of PREVACARE* Skin Care Products from Johnson \& Johnson Medical.

To learn more about PREVACARE* S Surgical Scrub or the complete line of PREVACARE* Skin Care Products, please call 1-888-633-7553. Or contact your Johnson \& Johnson Medical representative.
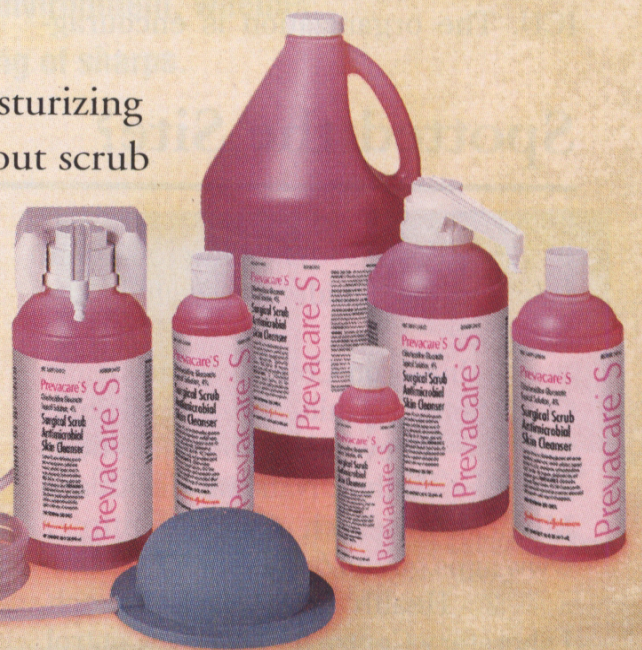